\title{
An RMHD study of transition between prompt and afterglow GRB phases
}

\section{Petar Mimica*}

Department for Astronomy and Astrophysics, University of Valencia

E-mail: petar.mimica@uv.es

\section{Dimitrios Giannios}

Max-Planck Institute for Astrophysics

E-mail: giannios@mpa-garching.mpg.de

\section{Miguel-Angel Aloy}

Department for Astronomy and Astrophysics, University of Valencia

E-mail: miguel.a.aloy@uv.es

We study the afterglow phases of a GRB through relativistic magnetohydrodynamic simulations. The evolution of a relativistic shell propagating into a homogeneous external medium is followed. We focus on the effect of the magnetization of the ejecta on the initial phases of the ejecta-external medium interaction. In particular we are studying the condition for the existence of a reverse shock into the ejecta, the timescale for the transfer of the energy from the shell to the shocked medium and the resulting multiwavelength light curves. To this end, we have developed a novel scheme to include non-thermal processes which is coupled to the relativistic magnetohydrodynamic code MRGENESIS in order to compute the non-thermal synchrotron radiation.

Supernovae: lights in the darkness

October 3-5, 2007

Maó (Menorca)

\footnotetext{
* Speaker.
} 


\section{Introduction}

Gamma ray bursts (GRBs) are believed to be the result of the internal energy release in an ultra-relativistic flow. The mechanisms responsible for launching and accelerating the flow remain poorly understood. Two alternatives for the production of the outflow have been extensively studied. Neutrino annihilation in the polar region of a hyperaccreting compact object may be the process which leads to the formation of a fireball, i.e. a flow dominated by thermal energy [17, 29, 34, 3, 4, 6]. Alternatively, if magnetic fields are powerful enough and have the appropriate topology, they can efficiently extract the rotational energy from the central engine (possibly an accretion disk [8], a rotating black hole [9] or a millisecond magnetar [33]) and launch a Poyntingflux dominated flow.

In order to achieve relativistic velocities, GRB outflows have to be launched with high energyto-mass ratio. In the fireball model, the acceleration of the fireball is caused by the internal pressure gradient, whereby thermal energy is converted into kinetic energy of the flow. At the end of the acceleration phase faster parts of the flow may collide with slower ones leading to internal shocks (i.e., shocks within the fireball ejecta) which power the GRB prompt emission [30, 11, 26, 27]. After the internal shock phase is over, the flow expands and cools before it enters the afterglow phase. In fireball models, the flow is expected to be at most weakly magnetized at the onset of the afterglow.

In many magneto-hydrodynamic (MHD) models for a GRB the flow is assumed to be Poyntingflux dominated (PDF) ${ }^{1}$. The acceleration of the PDF flow depends on the field geometry and the dissipation processes. Magnetic dissipation can convert Poynting flux into kinetic energy [12, 14] and also power the GRB prompt emission [23, 13, 15]. Different studies of MHD jet acceleration show that the magnetic energy is not completely converted into kinetic energy at the end of the acceleration phase. As a result, at large distances from the central engine magnetic energy of the flow may be comparable to the kinetic energy of the baryons [14] or even much larger [23, 32].

Fireball and PDF models predict weakly and strongly magnetized flow at the onset of the afterglow phase, respectively. The initial phases of the interaction of the GRB flow with the (circumburst) external medium depend on the strength of the magnetic fields in the flow. A particularly promising probe of the magnetization of the GRB flow (and, ultimately, of the mechanism responsible for a GRB) can thus come from understanding the early afterglow emission [20, 35, 16.

In this paper we outline the status of the ongoing numerical study of the interaction of magnetized ejecta with the external medium. In Sec. 2 we outline the analytical treatment of the ejectamedium interaction, while in Sec. 3 we give an overview of numerical methods and presents preliminary results of numerical simulations of such interaction. Summary is given in Sec. 4 .

\section{Ejecta-medium interaction}

We consider a homogeneous shell expanding into an external medium of constant density ${ }^{2}$. At large distances from the central engine (typically $R_{0} \approx 10^{15}-10^{17} \mathrm{~cm}$ ) a substantial interaction

\footnotetext{
${ }^{1}$ The initial magnetization of the flow may be affected by the pair-loading caused by the $v \bar{v}$-annihilation near the central engine 22 . 5 .

${ }^{2}$ Similar analysis can be performed for the wind profile where the density of the external medium scales as $r^{-2}$.
} 
begins, whereby the ejecta begins to decelerate due to the accumulation of the external material. We assume that at these distances the flow has already been accelerated and collimated. The internal dissipation mechanism, presumably responsible for the prompt emission (e.g., internal shocks, magnetic dissipation) is also expected to take place at a shorter distance from the central engine than the afterglow phase. After the internal dissipation is over, the flow expands and cools. Since we are interested in the afterglow phase, the shell is assumed to be cold. We denote the shell Lorentz factor by $\gamma_{0} \gg 1$ and its width by $\Delta_{0}$. In a radially expanding outflow the magnetic field component perpendicular to the direction of motion drops as $r^{-1}$, while the component in the direction of motion drops as $r^{-2}$, so that we expect the magnetic field to be dominated by the perpendicular component. We define the magnetization parameter as

$$
\sigma_{0}:=\frac{E_{P}}{E_{K}}=\frac{B_{0}^{2}}{4 \pi \gamma_{0} \rho_{0} c^{2}},
$$

where $E_{P}$ and $E_{K}$ are Poynting and kinetic energies in the shell, $\rho_{0}$ and $B_{0}$ its density and magnetic field measured in the central engine frame, respectively. With this definition a fireball corresponds to $\sigma_{0} \ll 1$ while a PDF has $\sigma_{0} \geq 1$. $c$ is the speed of light. The total energy of the shell is

$$
E=4 \pi R_{0}^{2} \Delta_{0}\left(\gamma_{0} \rho_{0} c^{2}+B_{0}^{2} / 4 \pi\right)=E_{K}\left(1+\sigma_{0}\right) .
$$

From Eqs. 2.1 and 2.2 we can see that $\sigma_{0}$ parametrizes the fraction of the total energy in the form of kinetic $\left(1 /\left(1+\sigma_{0}\right)\right)$ or of magnetic $\left(\sigma_{0} /\left(1+\sigma_{0}\right)\right)$ energy.

We first discuss the ejecta-medium interaction for non-magnetized ejecta, and then turn to the arbitrarily magnetized case. We also focus on the conditions for the existence of a reverse shock into ejecta of arbitrary magnetization.

\subsection{The case of $\sigma_{0} \ll 1$}

The evolution of the interface between the cold unmagnetized shell and the external medium is well understood. It was studied in detail analytically [31, 25], as well as using one-dimensional [19] and two-dimensional [10, 18, 24] numerical simulations.

At the interface between the shell and the ambient medium two shocks form, the forward shock propagating into the external medium, and the reverse shock propagating into the shell. Shocked shell and external medium are separated by the contact discontinuity. The forward shock is always ultra-relativistic, while the strength of the reverse shock depends on the density contrast between the shell and the external medium and the bulk Lorentz factor $\gamma_{0}$. We distinguish between relativistic and Newtonian reverse shocks [31]. The critical parameter differentiating both regimes is

$$
\xi:=l^{1 / 2} \Delta_{0}^{-1 / 2} \gamma_{0}^{-4 / 3},
$$

where $l=\left(3 E / 4 \pi n_{e} m_{p} c^{2}\right)^{1 / 3}$ is the Sedov length, $n_{e}$ the external medium number density, and $m_{p}$ the proton mass. In the Newtonian case $(\xi \gg 1)$ the shock is non-relativistic in the shell rest frame and does not decelerate the ejecta much, rather the ejecta decelerate once they accumulate a mass $\gamma_{0}^{-1}$ times their own mass from the external medium. In the relativistic case $(\xi \ll 1)$ the shock crosses the ejecta quickly and slows them down considerably. After the reverse shock crosses the ejecta, its non-linear interaction with the rear edge of the ejecta yields a number small shocks 
and rarefaction waves which cross the ejecta [31]. At later stages the evolution of the ejecta only depends on their total energy and the external medium density [7].

\subsection{The case of arbitrary $\sigma_{0}$}

The dynamics of magnetized ejecta has not been studied as thoroughly as that of unmagnetized ejecta. A qualitative difference from the unmagnetized case is that later evolutionary phases are influenced by the internal evolution of the magnetized shell. The initial phase of the evolution has recently been studied [36] by solving the ideal MHD shock conditions for arbitrarily magnetized ejecta with toroidal field. In particular, the dynamics of shock crossing has been studied assuming that there is a reverse shock. In that case, the reverse shock crosses the shell faster the higher the magnetization is. However, as we have recently showed [16], it is not always the case that a reverse shock forms.

\subsection{Conditions for the existence of a reverse shock}

The interaction of cold, non-magnetized ejecta with the external medium always produces a reverse shock which crosses the ejecta. This is the case since the ejecta is cold and, thus, the sound speed is small, which inhibits a fast transfer of the information ${ }^{3}$ of the interaction with the external medium throughout their volume. On the other hand, in a flow that is strongly magnetized and sub-fast magnetosonic (as in the Lyutikov \& Blandford 2003 model [23]) there is no reverse shock forming. The flow adjusts gradually to the changes of the pressure in the contact discontinuity that separates the magnetized flow from the shocked external medium. In [16] we generalize to arbitrarily magnetized ejecta and derive the condition for the formation of a reverse shock.

After a detailed treatment of this problem, we arrive to the following condition for the formation of a reverse shock [16]

$$
\xi<\frac{1}{\left(4 \sigma_{0}\right)^{1 / 3}}
$$

which can be rewritten in terms of shell parameters as ${ }^{4}$.

$$
\sigma_{0}<0.6 n_{0}^{1 / 2} \Delta_{12}^{3 / 2} \gamma_{2.5}^{4} E_{53}^{-1 / 2}
$$

Figure 1 (taken from [16]) shows the division of $\xi-\sigma_{0}$ parameter space in two regions, one where a reverse shock forms and another where its formation is suppressed. We note that, from the conditions in Eqs. 2.4 and 2.5, it follows that even for mildly magnetized shells a reverse shock can be suppressed. This indicates that the paucity of the observed optical flashes in GRB afterglows (associated with the reverse shock emission) may be caused by the suppression of the shock in many GRBs.

\section{Numerical simulations}

The main goal of this paper is to show the status of the ongoing numerical simulations performed and planed to test the theoretical developments devised by Giannios, Mimica \& Aloy [16].

\footnotetext{
${ }^{3}$ We remind the reader that the transfer of information in a fluid is channeled along its characteristic lines, which move with a speed $\lambda_{ \pm}=\left(v \pm c_{\mathrm{S}}\right)\left(1 \pm v c_{\mathrm{S}}\right)^{-1}$, where $c_{\mathrm{s}}$ and $v$ are the local sound speed and fluid velocity, respectively.

${ }^{4}$ We use the convention that $A=A_{x} 10^{x}$
} 


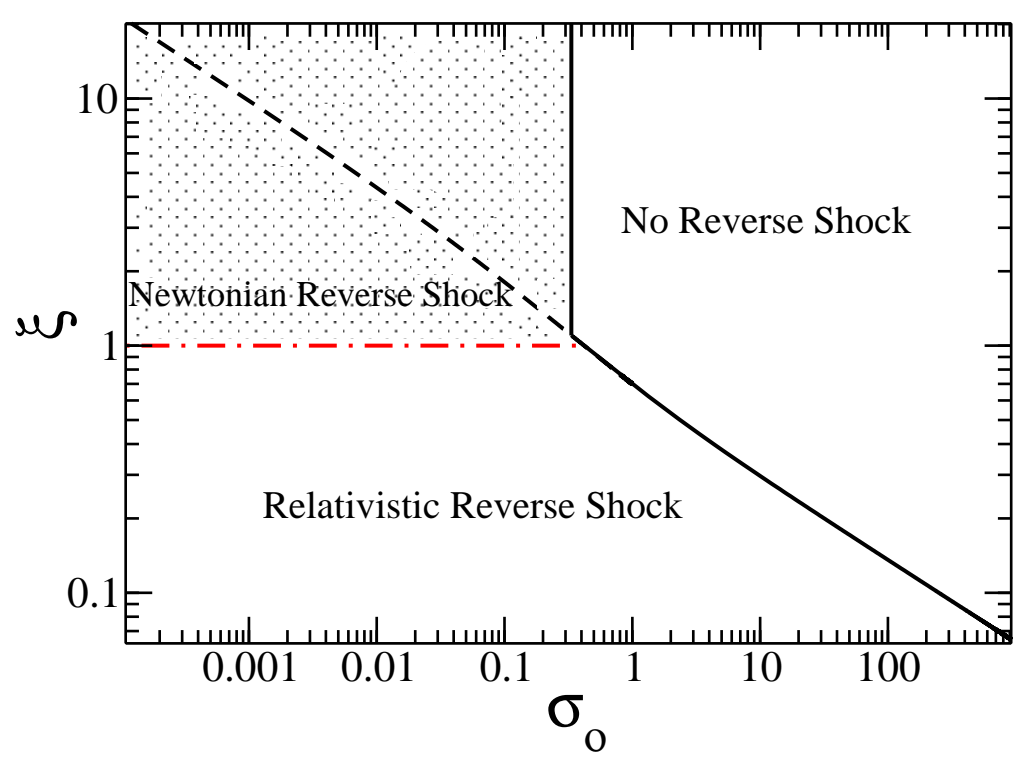

Figure 1: Existence of a reverse shock in the $\xi-\sigma_{0}$ parameter space. The dashed black line delimits regions where a reverse shock forms from the region where there is no reverse shock, ignoring the radial shell spreading. The solid black line shows the delimitation when the shell spreading is taken into account. See [16] for details.

More precisely, the reasons and motivation for performing numerical simulations of shell-ejecta interactions are:

1. Verification of the analytic approach: The results of the analytic work described in Sec. 2 , especially in Sec. 2.3, need to be verified by means of numerical simulations. We note that the line dividing the regions of formation and suppression of the reverse shock in Fig. 1, given by Eq. 2.4 is approximate, and it is necessary to perform numerical simulations for models whose initial parameters lay in the vicinity of the line.

2. Dynamics of shock propagation: We want to use numerical 4 models to study the influence of the magnetization on the propagation of the reverse shock through the shell. Snapshots of the interaction with the external medium of a unmagnetized $\left(\sigma_{0}=0\right)$, and a magnetized shell $\left(\sigma_{0}=1\right.$; Fig. 3) are shown in Figs. 2 and 3, respectively. Both snapshots correspond to the the same evolutionary time. It can be seen that, in the magnetized case (Fig. 3), the reverse shock has penetrated the shell deeper than in the unmagnetized case (Fig. 2). Our goal is to perform a parametric study where we aim to understand the propagation of shocks and rarefactions through the shell for different combinations of $\xi$ and $\sigma_{0}$.

3. Long term evolution and energy content: One of the important questions that simulations can answer is the timescale of the transfer of energy from the shell to the shocked external medium. Long-term numerical calculations are needed to determine the dependence of the 


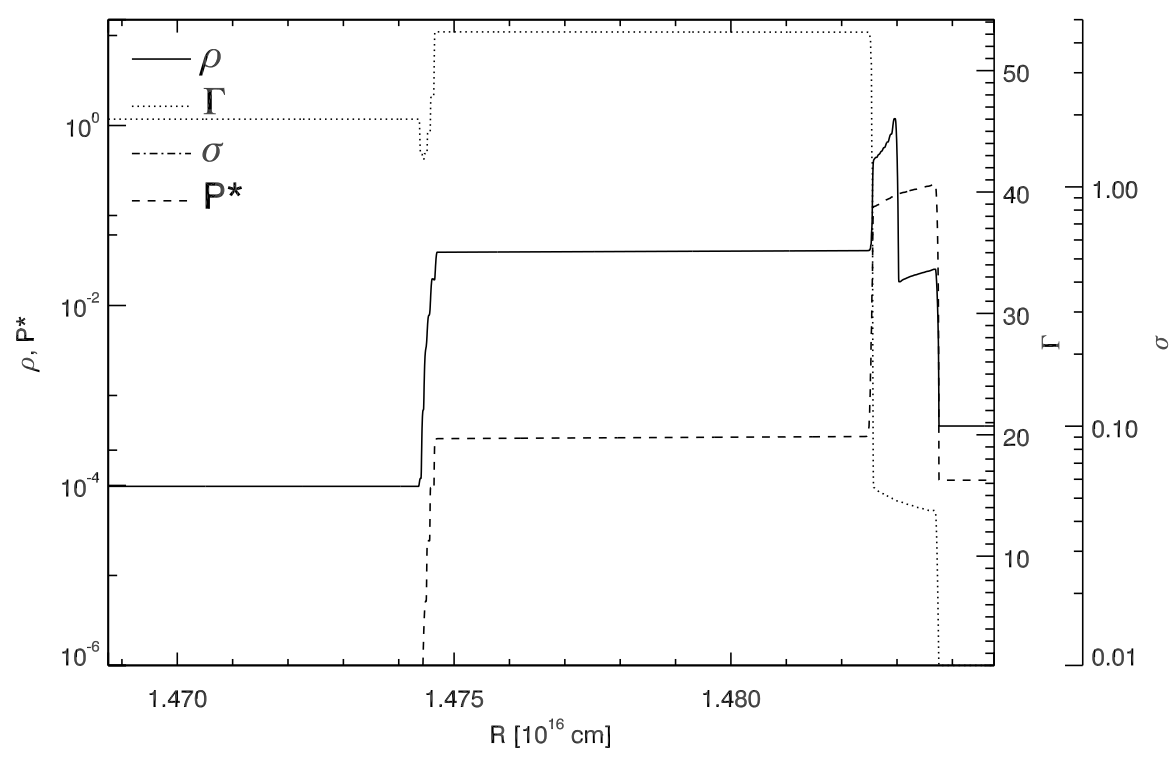

Figure 2: Snapshot from relativistic hydrodynamical simulation of a spherical, non-magnetized $\left(\sigma_{0}=0\right)$ shell that decelerates interacting with external medium of density $10 \mathrm{~cm}^{-3}$. The total energy of the shell is $E=10^{51} \mathrm{erg}$, its initial width $\Delta_{0}=10^{14} \mathrm{~cm}$ and bulk Lorentz $\gamma_{0} \simeq 50 . P^{*}$ (dashed line), $\rho$ (solid line) stand for the gas pressure and (lab frame) density respectively (in arbitrary units). With increasing radius, one can clearly see the reverse shock, contact discontinuity and forward shock located at $r \sim 1.483 \cdot 10^{16} \mathrm{~cm}$.

efficiency of the energy transfer on magnetization. We also want to investigate the long-term evolution of the blast wave and determine when the shell profile relaxes to the BlandfordMcKee solution [7].

4. Light curves: Finally, we wish to compute synthetic multi-wavelength afterglow light curves. Light curves are very sensitive to the magnetic field topology and strengh in the shell, shock strength and, especially, detailed radial profile $\gamma(R)$ of the Lorentz factor as the shell propagates into the external medium. They also depend on the distribution of shock accelerated particles. To see why $\gamma(R)$ is crucial for the light curve, consider the difference in the arrival time at the observer of signals emitted simultaneously from two points with radii $R$ and $R+\mathrm{d} R$, respectively. It turns out that the difference is $\mathrm{d} t \approx \mathrm{d} R \gamma(R)^{-2}$ for a relativistic shell. As it can be seen, a sudden drop of the Lorentz factor in one model as compared to the other will produce features which have longer observed duration. The resulting light curves of the two models can be very different. High-resolution simulations are needed to resolve sufficiently short time intervals in order to be able to study the influence of the magnetization on $\gamma(R)$ and the corresponding light curve.

We have developed a relativistic magnetohydrodynamic code MRGENESIS [28, 26, 27] based on a finite-volume, high-resolution, shock-capturing scheme GENESIS [1, 2, 21], which solves for the conservation laws of relativistic magnetohydrodynamics, and a module which follows the transport, evolution, and radiation from non-thermal particles. For the purpose of analyzing the 




Figure 3: Same as Fig. 2, but for the magnetized $\left(\sigma_{0}=1\right)$ shell. Note that the reverse shock crosses the ejecta faster with respect to the non-magnetized case in agreement with analytical expectations.

dynamics and the emission from afterglow shells, a very high resolution is needed. Numerical convergence tests showed that, in order to sufficiently resolve the shell, we need to resolve scales of the order of $\Delta_{0} \gamma_{0}^{-2}$. Thus, we need to cover the shell with, at least, $\gamma_{0}^{2}$ numerical zones. We use a grid re-mapping procedure [28], which can be thought of as a guided mesh refinement centered on the shell. Even with this procedure, the actual number of zones for a typical model is expected to be several millions. We are performing simulations on supercomputers of the Spanish Supercomputing Network.

\section{Conclusions}

We are performing high-resolution numerical studies of the transition from the prompt to the early afterglow phase of GRBs. The afterglow is modeled as the radiation from the relativistic shell expanding into the homogeneous external medium. We study the difference between the fireball (unmagnetized shell) and the Poynting-flux dominated (magnetized shell) models.

In the context of the early optical afterglow, we show analytically that even a moderate magnetization of the flow can suppress the existence of a reverse shock, and thus explain the apparent paucity of the optical flashes for a large number of early afterglows. We are currently performing simulations to study the formation and suppression of relativistic shocks in detail. To study the later phases of the afterglow we aim to determine the influence of the initial shell magnetization on the energy content, transfer of energy from the shell to the forward shock, and the long-term flow structure.

We have developed a novel scheme for treating non-thermal processes in relativistic magnetohydrodynamic simulations. This scheme is used to compute multi-wavelength light curves from 
numerical simulations. The aim is to study the influence of the initial magnetization on the shortand long-term light curves.

\section{Acknowledgements}

PM is at the University of Valencia with a European Union Marie Curie Incoming International Fellowship (MEIF-CT-2005-021603). MAA is a Ramón y Cajal Fellow of the Spanish Ministry of Education and Science. He also acknowledges partial support from the Spanish Ministry of Education and Science (AYA2004-08067-C03-C01, AYA2007-67626-C03-01, CSD2007-00050). The authors thankfully acknowledge the computer resources, technical expertise and assistance provided by the Barcelona Supercomputing Center - Centro Nacional de Supercomputación.

\section{References}

[1] M. A. Aloy, J. M. Ibáñez, J. M. Martí, E. Müller, GENESIS: A High-Resolution Code for Three-dimensional Relativistic Hydrodynamics, ApJ Suppl. Ser. (1999) 122, 151

[2] M. A. Aloy, J. A. Pons, J. M. Ibañez, An efficient implementation of flux formulae in multidimensional relativistic hydrodynamical codes, Comp. Phys. Comm. (1999) 120, 115

[3] M. A. Aloy, E. Müller, J. M. Ibañez, J. M. Martí, A. MacFayden, Relativistic jets from collapsars, ApJ (2000) 531, L119

[4] M. A. Aloy, H.-Th. Janka, E. Müller, Relativistic outflows from remnants of compact object mergers and their viability for short gamma-ray bursts, $A \& A$ (2005) 436, 273

[5] M. A. Aloy, M. Obergaulinger, Relativistic Outflows in Gamma-Ray Bursts, arXiv:astro-ph/0701187

[6] R. Birkl, M. A. Aloy, H. -Th. Janka, E. Müller, Neutrino pair annihilation near accreting, stellar-mass black holes, A\&A (2007) 463, 51

[7] R. D. Blandford, C. F. McKee, Fluid dynamics of relativistic blast waves, PhFl (1976) 19, 1130

[8] R. D. Blandford, D. G. Payne, Hydromagnetic flows from accretion discs and the production of radio jets, MNRAS (1982) 199, 983

[9] R. D. Blandford, R. L. Znajek, Electromagnetic extraction of energy from Kerr black holes, MNRAS (1977) 179, 433

[10] J. K. Cannizzo, N. Gehrels, E. T. Vishniac, A Numerical Gamma-Ray Burst Simulation Using Three-Dimensional Relativistic Hydrodynamics: The Transition from Spherical to Jetlike Expansion, ApJ (2004) 601, 380

[11] F. Daigne, R. Mochkovitch, Gamma-ray bursts from internal shocks in a relativistic wind: temporal and spectral properties, MNRAS (1998) 296, 275

[12] G. Drenkhahn, H. C. Spruit, Efficient acceleration and radiation in Poynting flux powered GRB outflows, $A \& A$ (2002) 391, 1141

[13] D. Giannios, Prompt emission spectra from the photosphere of a GRB, A\&A (2006) 457, 763

[14] D. Giannios, H. C. Spruit, The role of kink instability in Poynting-flux dominated jets, A\&A (2006) 450, 887 
[15] D. Giannios, H. C. Spruit, Spectral and timing properties of a dissipative gamma-ray burst photosphere, A\&A (2007) 469, 1

[16] D. Giannios, P. Mimica, M. A. Aloy, On the existence of a reverse shock in magnetized GRB ejecta, $A \& A$ (2008) 478, 747

[17] J. Goodman, Are gamma-ray-bursts optically thick?, ApJ (1986) 308, L47

[18] J. Granot, M. Miller, T. Piran, W. M. Suen, P. A. Hughes, Light Curves from an Expanding Relativistic Jet, in proceedings of Gamma-ray Bursts in the Afterglow Era (2001), 312

[19] S. Kobayashi, T. Piran, R. Sari, Hydrodynamics of a Relativistic Fireball: The Complete Evolution, ApJ (1999) 513, 669

[20] P. Kumar, A. Panaitescu, A unified treatment of the gamma-ray burst 021211 and its afterglow, MNRAS (2003) 346, 905

[21] T. Leismann, L. Antón, M. .A. Aloy, E. Müller, J. M. Martí, J. A. Miralles, J. M. Ibáñez, Relativistic MHD simulations of extragalactic jets, A\&A (2005) 436, 503

[22] A. Levinson, D. Eichler, Baryon Purity in Cosmological Gamma-Ray Bursts as a Manifestation of Event Horizons , ApJ (1993) 418, 386

[23] M. Lyutikov, R. D. Blandford, Gamma Ray Bursts as Electromagnetic Outflows, arXiv arXiv:astro-ph/0312347

[24] Z. Meliani, R. Keppens, F. Casse, D. Giannios, AMRVAC and relativistic hydrodynamic simulations for gamma-ray burst afterglow phases, MNRAS (2007) 376, 1189

[25] P. Mészáros, M. J. Rees, Optical and Long-Wavelength Afterglow from Gamma-Ray Bursts, ApJ (1997) 476, 232

[26] P. Mimica, M. A. Aloy, E. Müller, W. Brinkmann, Which physical parameters can be inferred from the emission variability of relativistic jets?, A\&A (2005) 451, 103

[27] P. Mimica, M. A. Aloy, E. Müller, Internal shocks in relativistic outflows: collisions of relativistic shells, A\&A (2007) 466, 93

[28] P. Mimica, M. A. Aloy, E. Müller, W. Brinkmann, Synthetic X-ray light curves of BL Lacs from relativistic hydrodynamic simulations, $A \& A$ (2004) 418, 947

[29] B. Paczyński, Gamma-ray bursters at cosmological distances, ApJ (1986) 308, L43

[30] M. J. Rees, P. Mészáros, Unsteady outflow models for cosmological gamma-ray bursts, ApJ (1994) 430, L93

[31] R. Sari, T. Piran, Hydrodynamic Timescales and Temporal Structure of Gamma-Ray Bursts, ApJ (1995) 455, L143

[32] C. Thompson, Deceleration of a Relativistic, Photon-rich Shell: End of Preacceleration, Damping of Magnetohydrodynamic Turbulence, and the Emission Mechanism of Gamma-Ray Bursts, A\&A (2006) $\mathbf{6 5 1}, 333$

[33] V. V. Usov, Millisecond pulsars with extremely strong magnetic fields as a cosmological source of gamma-ray bursts, Nature (1992) 357, 472

[34] S. W. Woosley, Gamma-ray bursts from stellar mass accretion disks around black holes, ApJ (1993) 405, 273 
[35] B. Zhang, S. Kobayashi, P. Mészáros, Gamma-Ray Burst Early Optical Afterglows: Implications for the Initial Lorentz Factor and the Central Engine, ApJ (2003) 595, 950

[36] B. Zhang, S. Kobayashi, Gamma-Ray Burst Early Afterglows: Reverse Shock Emission from an Arbitrarily Magnetized Ejecta, ApJ (2005) 628, 315 